Original Article Journal of Epilepsy Research pISSN 2233-6249 / eISSN 2233-6257

Received February 22, 2018

Revised July 25, 2018

Accepted December 5, 2018

Corresponding author:

Ki Joong Kim, MD, PhD

Division of Pediatric Neurology, Departmen of Pediatrics, Pediatric Clinical Neuroscience Center, Seoul National University Children's Hospital, Seoul National University College of Medicine, 101 Daehak-ro Jongno-gu, Seou 03080, Korea

Tel. $+82-2-2072-3367$

Fax. +82-2-743-3455

E-mail; pednr@snu.ac.kr

\title{
Clinical Experience with Perampanel in Intractable Focal Epilepsy Over 12 Months of Follow-Up
}

\author{
Soo Yeon Kim, MD', Woo Joong Kim, MD', Hyuna Kim, MD', Sun Ah Choi, MD², \\ Byung Chan Lim, MD, PhD', Jong-Hee Chae, MD, PhD', Ki Joong Kim, MD, PhD' \\ ${ }^{1}$ Department of Pediatrics, Pediatric Clinical Neuroscience Center, Seoul National University Children's Hospital, \\ Seoul; ${ }^{2}$ Department of Pediatrics, Seoul National University Bundang Hospital, Seongnam, Korea
}

\begin{abstract}
Background and Purpose: There are only limited studies on perampanel (PER), one of the latest antiepileptic drug. This study aimed to evaluate the long-term efficacy and tolerability of perampanel as an add-on therapy in patients with intractable focal epilepsy.
\end{abstract}

Methods: The medical records of 97 patients (age, 12-30 years) were retrospectively reviewed and analyzed. The patients had been diagnosed with focal epilepsy, treated with PER, and regularly followed up over 12 months. Results: All patients had uncontrolled seizures despite treatment with two or more antiepileptic drugs. The mean age of seizure onset was 5.2 years (range, 0-17.0). PER was first prescribed at an average age of 15.7 years (range, 12.0-25.3), and mean follow-up duration after PER initiation was 15.9 months (range, 12-20). The responder rate was $41.7 \%$, with over $75 \%$ seizure reduction obtained in 11 cases $(15.3 \%)$, including three seizure-free cases (4.2\%). The retention rates at 3, 6, 12, and 18 months of follow-up were $82.5 \%$ (80/97), 72.1\% (70/97), 60.8\% (59/97), and 37.5\% (6/16), respectively. Forty-four patients (44/97, $45.4 \%$ ) discontinued PER, because of treatment-related adverse events in 20 (20.6\%) and no efficacy in $24(24.7 \%)$. Treatment-related adverse events were reported by 52 patients (53.6\%). The most common adverse event was somnolence or lethargy, reported by 17 patients $(17 / 97,23 \%)$, followed by dizziness $(15 / 97,20 \%)$ and psychological problems such as aggressiveness or irritability $(15 / 97,20 \%)$. Thirty-three patients $(33 / 52,63.4 \%)$ showed their first adverse symptom for 2 or $4 \mathrm{mg} / \mathrm{day}$ of PER.

Conclusions: PER would be an effective therapeutic option for patients with intractable focal epilepsy. However, careful monitoring of adverse events is essential from treatment initiation, with particular attention to psychological problems in adolescents and young adults. (2018;8:61-65)

Key words: Perampanel, Intractable seizure, Focal epilepsy, Efficacy, Tolerability

\section{Introduction}

Despite ongoing investigations of epilepsy and the development of several new antiepileptic drugs (AEDs), about a third of patients remain intractable. ${ }^{1,2}$ Recent exploration of new AEDs has focused on novel molecular targets, such as non-neuronal cells or neurotransmitter receptors, due to the strength of their effects on seizures and the role of para-neuronal cells in epilepsy. ${ }^{3-5}$ Perampanel (PER) is one of the newest drugs that targets glutamate excitatory neurotransmission through an $\alpha$-amino-3-hydroxy-5-methyl-4-isoxazolepropinonic acid receptor. It is considered a better target than other glutamate-related receptors, because it contributes to fast synaptic excitation at the seizure onset. ${ }^{6}$ In a phase III clinical trial, PER showed a fair level of response rate among patients with focal and primary tonic-clonic seizures. Treatment-related adverse events were reported in more than $70 \%$ of patients taking $8 \mathrm{mg}$ or higher dose of PER, but most of these were not severe. ${ }^{7-9}$ In particular, the responder rate was maintained during a 2-year follow-up extension study. ${ }^{10}$ Several clinical reports have been published since its release, but the efficacy profiles vary between studies from $27 \%$ to $53 \%$ and reported adverse events have been diverse and differ among age groups. Considering the diversity of patient ages and epileptic syndromes included, long-term pediatric data have been limited. ${ }^{11-13}$ Thus, we report our experiences with PER in adolescents and young adults with intractable focal epilepsy. 


\section{Methods}

The study was approved by the Institutional Review Board (IRB) of Seoul National University Hospital (IRB No. 1802-052-921). We enrolled patients with intractable focal epilepsy, who were started on PER and had regular check-ups over 12 months at Seoul National University Children's Hospital. Intractability was defined as failure to control seizures with adequate trials of two or more AEDs. Patients with generalized or undetermined seizure were excluded. We reviewed medical records retrospectively, including age, sex, seizure onset age, personal history of AEDs, results of laboratory and imaging tests, seizure frequency before and after PER administration, and newly occurred adverse events during PER treatment. Efficacy was evaluated based on the proportion of patients who were responders (i.e., who showed $50 \%$ or more seizure reduction), comparing the average number of seizures during the 28 days between baseline and maintenance periods. The baseline period was defined as the 12 weeks prior to first PER administration, and AEDs were maintained at the same dose during this period. The maintenance period was defined as total treatment duration after completion of 16 weeks of PER titration, although the actual titration schedule varied every 4-8 weeks in $71 \%$ of patients, and every 2-3 weeks in 20\% (remaining patients discontinued PER at $2 \mathrm{mg} /$ day). The final dose of PER was determined by the physician, considering the efficacy and tolerability. Consequently, efficacy data were obtained from patients consuming PER for more than 16 weeks, the titration period by definition. Tolerability was evaluated based on the retention rate and profile of adverse events. The retention rate at 3,6, 12, and 18 months was calculated, and all patients were asked about any adverse events, including dizziness, ataxia, somnolence, fall, seizure aggravation, weight change, and psychological problems during PER use at every outpatient clinic visit. Psychological adverse events included aggression, behavioral problem, excessive irritability, suicidal idea, and significant mood changes. Statistical analyses were conducted using IBM SPSS Statstics 22 software suite (IBM SPSS, Chicago, IL, USA) with Student's $t$-test for continuous variables and Pearson's chi-square test for categorical variables. Statistical significance was considered at $p<0.05$.

\section{Results}

\section{Patient characteristics}

Demographic data of the 97 enrolled patients are presented in
Table 1. The first seizure occurred at an average age of 5.2 years (range, 0-15.4), and patients were treated with AEDs for approximately 13 years (range, 1.5-25.4 years). Forty patients $(41.2 \%)$ were prescribed PER for the first time before 18 years of age, while the others (58.8\%) began PER between 18-30 years of age. The mean follow-up duration after PER treatment was 15.9 months (range, 12-20 months) with a mean duration of treatment with PER of 10 months (range, 0-19 months). All patients had focal epilepsy with variable etiology. One-third of the patients (36.1\%) had cryptogenic focal epilepsy without any structural lesion detected on the brain magnetic resonance imaging, whereas the others $(63.9 \%)$ had structural lesion including congenital anomaly and acquired injury. The most common structural abnormality was focal cortical dysplasia and post-infectious lesion. Enrolled patients typically had highly intractable seizure. About half the patients $(45,46.4 \%)$ were on four or

Table 1. Demographic data for all patients

\begin{tabular}{|c|c|}
\hline & Value $(n=97)$ \\
\hline Sex ratio (male : female) & $62: 35$ \\
\hline Total follow-up duration (years) & $13.3(2.1-25.4)$ \\
\hline Age of seizure onset (years) & $5.2(0.0-17.0)$ \\
\hline Total treatment duration (years) & $15.7(4.3-25.3)$ \\
\hline \multicolumn{2}{|l|}{ Age at first perampanel dosing } \\
\hline $12-18$ years & $40(41.2)$ \\
\hline $18-30$ years & $57(58.8)$ \\
\hline Treatment duration with perampanel (months) & $9.8(0-19)$ \\
\hline $\begin{array}{l}\text { Follow-up duration after perampanel administration } \\
\text { (months) }\end{array}$ & $15.9(12-19)$ \\
\hline \multicolumn{2}{|l|}{ Seizure etiology } \\
\hline Cryptogenic & $35(36.1)$ \\
\hline \multicolumn{2}{|l|}{ Structural } \\
\hline Hippocampal sclerosis & $9(9.3)$ \\
\hline Other focal cortical dysplasia & $12(12.4)$ \\
\hline Tuberous sclerosis & $5(5.2)$ \\
\hline Other congenital malformation & $8(8.2)$ \\
\hline Post-infectious & $12(12.4)$ \\
\hline Perinatal hypoxic brain injury & $8(8.2)$ \\
\hline Others & $8(8.20)$ \\
\hline \multicolumn{2}{|l|}{ Number of concomitant antiepileptic drugs } \\
\hline 2 & $18(18.6)$ \\
\hline 3 & $34(35.1)$ \\
\hline 4 & $29(29.9)$ \\
\hline 5 & $11(11.3)$ \\
\hline$\geq 6$ & $5(5.2)$ \\
\hline \multicolumn{2}{|l|}{ Treatment other than antiepileptic drugs } \\
\hline Epilepsy surgery & $22(22.7)$ \\
\hline Vagus nerve stimulation & $14(14.3)$ \\
\hline
\end{tabular}

Values are presented as mean (range) or number (\%) unless otherwise indicated. 
more concomitant AEDs. Epilepsy surgery was conducted on 22 patients $(22.5 \%)$ and vagus nerve stimulation (VNS) was simultaneously used by 14 (14.3\%) during PER administration.

\section{Efficacy profiles and predictors}

Efficacy was analyzed using seizure reduction records in 72 patients who were prescribed PER for 16 or more weeks. Titration schedule varied depending on the patient, but usually was every 4 or 8 weeks. Mean maintenance dose of PER was $6.6 \mathrm{mg}$ (range, 2-12 $\mathrm{mg}$ ), and responder rate was $41.7 \%$ (30/72 patients) on PER treatment. Among these, over $75 \%$ of seizure reduction was achieved in 11 patients (15.3\%), including three seizure-free cases (4.2\%). Responders accounted for 16 of the 34 patients (47.1\%) in the adolescents group (ages 12-18 years) and 14 of 38 (36.8\%) in the young adults group (ages $18-30$ years). More than $50 \%$ seizure reduction was observed in nine out of 22 patients with cryptogenic focal epilepsy (40.9\%), while 21 of 50 patients (42.0\%) with structural lesions showed this rate of reduction. We compared clinical profiles of responders and non-responders to predict the fair efficacy of PER (Table 2). There were no significant clinical predictors of better response. However, higher number of concomitant medications, which indicated intractability of seizures, was likely to reduce the responder rate. The patients who achieved seizure-free status were 15.7, 19.1, and 22.4 years of age during PER initiation. All three patients had structural etiology with two or three concomitant AEDs. Their baseline seizure frequencies were 12, 10, and 0.7 times per 28 days, respectively, and their seizures decreased to zero with a maintenance dose of $4 \mathrm{mg} /$ day. All three patients maintained a seiz- ure-free status for over 12 months (one case for 14 months, others for 16 months).

\section{Retention rate and tolerability profiles}

The retention rates at $3,6,12$, and 18 months were $82.5 \%$ (80/97), 72.1\% (70/97), 60.8\% (59/97), and 37.5\% (6/16), respectively. Forty-four patients (44/97, 45.4\%) discontinued PER before their last visit. Among these, 20 patients (20/97, 20.6\%) stopped the medication due to adverse events, while others (24/97, $24.7 \%$ ) discontinued due to lack of efficacy. Fifty-two patients $(53.6 \%)$ reported 74 types of adverse events (Fig. 1A), with somnolence or lethargy being the most common and reported in 17 patients (17/97, 17.5\%). Dizziness and ataxia accounted for 15.3\% and $9.3 \%$ of total patients, respectively, where three patients fell and had related trauma because of severe dizziness or ataxia. Psychological problems such as aggression or excessive irritability were other major adverse events observed in 15 patients (28\%). Mood changes including depression or anxiety were not observed. Seizure aggravation was reported in five patients $(5 / 97,5.2 \%)$, who took PER for 0-3 months with a maximum dose of 4-6 mg/day. One of these patients showed a prolonged seizure few days after PER add-on. Over $60 \%$ of patients $(32 / 52,62 \%)$ reported their treatment-related adverse events at a PER dose of $4 \mathrm{mg} /$ day or less (Fig. 1B). In particular, 12 patients experienced symptoms at the initiation dose of 2 $\mathrm{mg} /$ day, while 17 patients who once had some adverse event maintained the PER until their last clinic visit with fair efficacy. Among them, four patients reported improvement of symptoms after dose reduction and the rest adjusted with the same dose.

Table 2. Univariate analysis of clinical predictors for responder rate

\begin{tabular}{|c|c|c|c|}
\hline & Responder $(n=30)$ & Non-responder $(n=42)$ & $p$-value \\
\hline Sex ratio (male : female) & $17: 13$ & $28: 14$ & 0.389 \\
\hline Seizure onset age (years) & $5.4( \pm 4.84)$ & $4.5( \pm 4.64)$ & 0.436 \\
\hline Total treatment duration (years) & $15.1( \pm 4.68)$ & $16.2( \pm 5.33)$ & 0.358 \\
\hline Mean baseline seizure frequency (times/28 days) & $31.6( \pm 62.13)$ & $20.1( \pm 53.06)$ & 0.407 \\
\hline Mean number of concomitant antiepileptic drugs & $3.23( \pm 0.97)$ & $3.76( \pm 1.27)$ & 0.065 \\
\hline Seizure etiology & & & 0.612 \\
\hline Cryptogenic & $9(30.0)$ & $13(31.0)$ & \\
\hline Structural & $21(70.0)$ & $29(69.0)$ & \\
\hline Age at first perampanel dosing & & & 0.381 \\
\hline $12-18$ years & $16(53.3)$ & $18(42.9)$ & \\
\hline $18-30$ years & $14(46.7)$ & $24(57.1)$ & \\
\hline Maintenance dose of perampanel (mg) & $6.5( \pm 2.39)$ & $6.8( \pm 2.46)$ & 0.551 \\
\hline Intellectual disability & $15(35.7)$ & $13(43.3)$ & 0.514 \\
\hline
\end{tabular}

Values are presented as number (\%) unless otherwise indicated. 
A

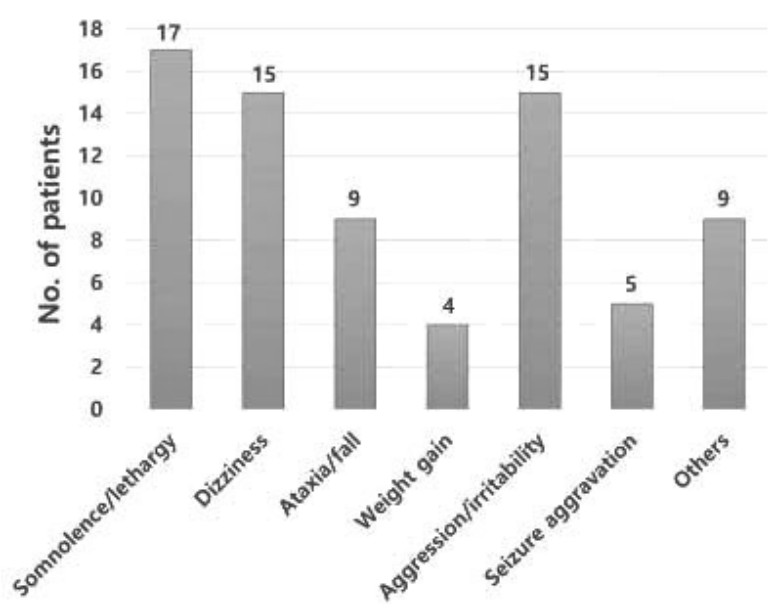

B

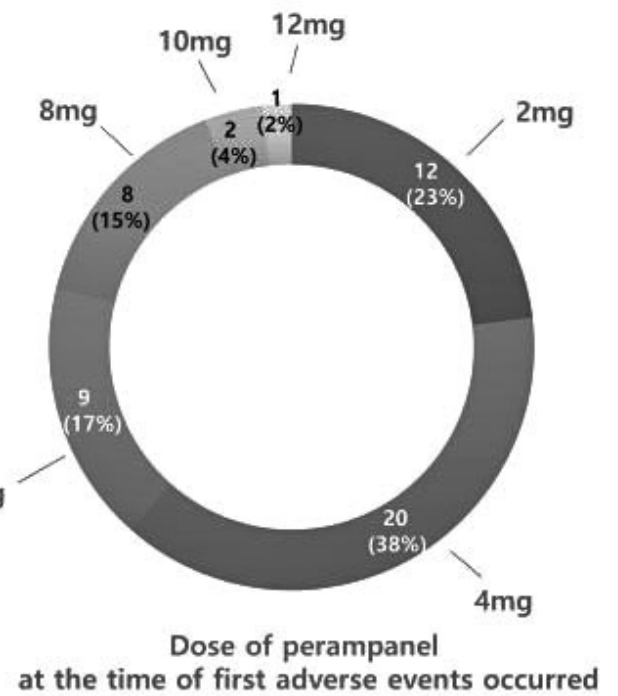

Figure 1. Profiles for adverse events that occurred during treatment with perampanel. (A) Detailed list and proportions for adverse events and (B) minimum dose of perampanel at which adverse events occurred.

\section{Discussion}

Our cohort represented patients with severely intractable epilepsy. This has been a rare characteristic in other cohorts, particularly of adolescents. ${ }^{12,14}$ About $50 \%$ of our patients were on four or more AEDs, and epilepsy surgery and VNS were performed in $22(22.7 \%)$ and 14 patients (14.4\%), respectively. Average seizure frequency during the baseline period was 26.3 per 28 days. Nevertheless, PER showed a fair level of efficacy with a responder rate of $41.7 \%$. According to previous real world retrospective studies, responder rates ranged from $19 \%$ to $50 \%$ and our data showed relatively high responder rate. ${ }^{11,14,15}$ Efficacy in the present cohort might be overestimated because 21 patients who discontinued PER within four months were excluded. Among these, however, 15 patients (71.4\%) discontinued the medication due to treatment-related adverse events regardless of effectiveness, and six were prescribed $2 \mathrm{mg} /$ day of PER until discontinuation. Therefore, any bias was most likely limited, and our data clearly supported the effectiveness of PER as an add-on therapy in patients with severely intractable focal seizures. As described in Table 2, neither intellectual disability nor age of PER initiation was associated with higher effectiveness. Other variables too did not show significant differences $(p<0.05)$, but the large number of adverse events were likely to have association with lower efficacy $(p<0.1)$. It represented intractability of seizures, which have been well known as an important factor for reducing the effectiveness of additive AED. Patients with four or more concomitant AEDs showed a $28.6 \%$ res- ponder rate, and PER could be one treatment option in these patients. Further studies of PER efficacy in patients with detailed epilepsy syndrome, etiology, or other combined conditions are needed.

The proportion of adverse events and related medication withdrawal varies with studies, even for similar age groups. ${ }^{12-14,16}$ In the present study, adverse events and related AED discontinuation accounted for $53.6 \%$ and $20 \%$ of the cohort, respectively, both of which were relatively high. We also recognize that many adverse events reported were during administration of a low dose. This was inconsistent with previously established relation between dose and side effects. ${ }^{9}$ The average dose of PER during adverse events was $3.9 \mathrm{mg} /$ day, despite the mean maximum dose of $6.9 \mathrm{mg} /$ day in the present study. Twelve patients $(12 / 52,23 \%)$ reported adverse events at $2 \mathrm{mg} / \mathrm{day}$ dosage within few days of PER initiation. We can infer from this that a high burden of concomitant AEDs influenced these events, and clinically it is clear that careful observation is necessary from the initial use of PER in patients with multiple AEDs. Among such adverse events, psychiatric problems including aggression, irritability, anxiety, and mood instability have been noted from PER clinical trials. These appear to be more frequent among younger populations and among patients with prior psychiatric problems. ${ }^{16-18}$ However, Snoeijen-Schouwenaars et al. ${ }^{19}$ have insisted that pre-existing behavioral problems or polypharmacy are unassociated with high psychiatric adverse events. In our study, 15 patients (15.5\%) showed aggression, irritability, or other behavioral problems at an average dose of $5.1 \mathrm{mg} /$ day. Nine patients presented symptoms at a dose of $4 \mathrm{mg}$ or less of PER. All except two patients finally 
discontinued the PER due to their adverse events. A 16-year-old male patient with three concomitant AEDs actually attempted suicide 2 months after PER administration ( $2 \mathrm{mg} /$ day dose) and was admitted to a psychiatric ward. His symptoms completely disappeared after PER discontinuation. Among the patients with psychiatric adverse events, three patients $(3 / 15,20 \%)$ had pre-existing psychiatric problems, whereas only six $(6 / 82,7.3 \%)$ in that group were without psychiatric adverse events, although this was not statistically significant ( $p=$ 0.193). Intellectual disability was noted in seven and 31 patients (46.7\% and $37.8 \%)$ who were with and without psychiatric adverse events, respectively $(p=0.462)$. Furthermore, age did not show any significant association with occurrence of adverse events $(p=0.471)$, although our cohort ranged from 12 to 30 years of age. The retention rate remained above $60 \%$ till 12 months of follow-up, but rapidly decreased to $37.5 \%$ at 18 months. In our cohort, all patients were monitored over 12 months, but 14 were monitored over 18 months after PER administration. Therefore, the retention rate at 18 months had low reliability and needed more follow-up. Our study also had some limitations that are commonly observed in other retrospective studies. The protocol for drug administration including initiation and titration schedule was not clearly established, and adverse events were identified by retrospective record reviews. Longer data collection in larger number of patients might be necessary for further validation of PER in clinical setting.

In conclusion, PER showed a fair level of efficacy in the present study, and it could be considered as a treatment option for intractable focal epilepsy. However, adverse events including psychiatric problems were very frequent even at a low dose. Therefore, pre-explanation about treatment-related adverse events and close monitoring at clinic visits is necessary.

\section{Conflicts of Interest}

All authors have no conflicts of interest to disclose.

\section{References}

1. Kwan P, Arzimanoglou A, Berg AT, et al. Definition of drug resistant epilepsy: consensus proposal by the ad hoc Task Force of the ILAE Commission on Therapeutic Strategies. Epilepsia 2010;51:1069-77.

2. Verrotti A, Loiacono G, Coppola G, Spalice A, Mohn A, Chiarelli F. Pharmacotherapy for children and adolescents with epilepsy. Expert Opin Pharmacother 2011;12:175-94.

3. Punnakkal P, Dominic D. NMDA receptor GluN2 subtypes control epileptiform events in the hippocampus. Neuromolecular Med 2018;20:90-6.
4. Zhao H, Lin Y, Chen S, Li X, Huo H. 5-HT3 Receptors: a potential therapeutic target for epilepsy. Curr Neuropharmacol 2018;16:29-36.

5. Steinhäuser C, Grunnet M, Carmignoto G. Crucial role of astrocytes in temporal lobe epilepsy. Neuroscience 2016;323:157-69.

6. Rogawski MA. Revisiting AMPA receptors as an antiepileptic drug target. Epilepsy Curr 2011;11:56-63.

7. French $J A$, Krauss $G L$, Biton $V$, et al. Adjunctive perampanel for refractory partial-onset seizures: randomized phase III study 304. Neurology 2012;79:589-96.

8. French JA, Krauss GL, Steinhoff BJ, et al. Evaluation of adjunctive perampanel in patients with refractory partial-onset seizures: results of randomized global phase III study 305. Epilepsia 2013;54:117-25.

9. Krauss GL, Serratosa JM, Villanueva V, et al. Randomized phase III study 306: adjunctive perampanel for refractory partial-onset seizures. Neurology 2012;78:1408-15.

10. Krauss GL, Perucca E, Ben-Menachem E, et al. Perampanel, a selective, noncompetitive $\alpha$-amino-3-hydroxy-5-methyl-4-isoxazolepropionic acid receptor antagonist, as adjunctive therapy for refractory partial-onset seizures: interim results from phase III, extension study 307. Epilepsia 2013;54:126-34.

11. Juhl S, Rubboli G. Perampanel as add-on treatment in refractory focal epilepsy. The Dianalund experience. Acta Neurol Scand 2016;134:374-7.

12. De Liso $P$, Vigevano $F$, Specchio $N$, et al. Effectiveness and tolerability of perampanel in children and adolescents with refractory epilepsies-An Italian observational multicenter study. Epilepsy Res 2016;127:93-100.

13. Rohracher $A$, Kalss $G$, Leitinger $M$, et al. Two-year real-world experience with perampanel in patients with refractory focal epilepsy: Austrian data. Ther Adv Neurol Disord 2016;9:445-53.

14. Biro A, Stephani U, Tarallo T, et al. Effectiveness and tolerability of perampanel in children and adolescents with refractory epilepsies: first experiences. Neuropediatrics 2015;46:110-6.

15. Singh K, Shah YD, Luciano D, Friedman D, Devinsky O, Kothare SV. Safety and efficacy of perampanel in children and adults with various epilepsy syndromes: a single-center postmarketing study. Epilepsy Behav 2016; 61:41-5.

16. Datta AN, Xu Q, Sachedina S, Boelman C, Huh L, Connolly MB. Clinical experience with perampanel for refractory pediatric epilepsy in one Canadian center. J Child Neurol 2017;32:834-9.

17. Rosenfeld W, Conry J, Lagae L, et al. Efficacy and safety of perampanel in adolescent patients with drug-resistant partial seizures in three double-blind, placebo-controlled, phase III randomized clinical studies and a combined extension study. Eur J Paediatr Neurol 2015;19:435-45.

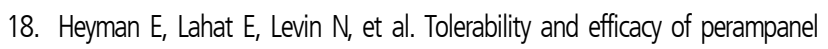
in children with refractory epilepsy. Dev Med Child Neurol 2017;59:441-4.

19. Snoeijen-Schouwenaars FM, van Ool JS, Tan IY, Schelhaas HJ, Majoie $\mathrm{MH}$. Evaluation of perampanel in patients with intellectual disability and epilepsy. Epilepsy Behav 2017;66:64-7. 